\title{
INDEPENDENT COMPONENT ANALYSIS BASED INCOHERENT TARGET DECOMPOSITIONS FOR POLARIMETRIC SAR DATA - PRACTICAL ASPECTS
}

\author{
Gabriel Vasile* \\ * Grenoble-Image-sPeach-Signal-Automatics Lab, GIPSA-lab \\ Univ. Grenoble Alpes, CNRS / Grenoble-INP, Grenoble, France \\ gabriel.vasile@grenoble-inp.fr
}

\begin{abstract}
The Independent Component Analysis (ICA) has been recently introduced as a reliable alternative to identify canonical scattering mechanisms within PolSAR images. This paper addresses an important practical aspect for applying such methods on real data, namely speckle filtering with ICA. A novel algorithm is introduced by adjusting the Lee's sigma filter to the particular nature of the Touzi's polarimetric decomposition. In its current form, it allows the use of the ICA mixing matrix in the derived speckle filter.
\end{abstract}

Index Terms - Independent Component Analysis, PolSAR, speckle filtering

\section{INTRODUCTION}

Polarimetric target decomposition is a PolSAR image interpretation technique that relies on the analysis of the interaction between the illuminated area and the transmitted waveform, considering each polarimetric state of the latter. More specifically, it enables the description of an image cell as a sum of canonical scattering mechanisms (also called as target vectors) making it more intuitive to understand the behaviour of the clutter and therefore to analyse it [1].

Polarimetric target decompositions are mainly classified in coherent, if their interest lies on the scattering matrix analysis for each resolution cell, like the ones proposed by Cameron [2], or incoherent, if they are based on a statistical analysis of neighbouring pixels. Incoherent target decomposition (ICTD) theory assumes that the scattering process in most natural media is a combination of coherent speckle noise and random vector scattering effects. Therefore, a stochastic approach is required and the concept of average or dominant scattering mechanisms is associated to each imaging cell [3]. Most methods described in the literature focus on the Hermitian, semidefinite positive coherence or covariance matrix [3]. Nevertheless, the investigation of higher order moments has recently sparked great interest of the SAR community, introducing supplementary information to the clutter analysis

Thanks to the French Aerospace Lab for providing the PolSAR data. and consequently leading to new ICTD approaches [4], [5], [6].

The combined use of the Eigenvector approach with Cloude and Pottier's parametrisation gave rise to one of the most employed and most traditional classification schemes in PolSAR data analysis, the $\mathrm{H} / \alpha$ feature space [7]. The entropy, $\mathrm{H}$, measures the degree of randomness of the scattering phenomenon, given as a function of the eigenvalues of the coherence matrix. Each eigenvector correspond to a scattering mechanism within the image cell and therefore each one will provide a different $\alpha$ angle. The authors in [7] state that the best estimate of such parameter to represent the image cell is an weighted average based on the eigenvalues of the coherence matrix.

The parameters $\mathrm{H}$ and $\alpha$ are plotted in a plane, originating the so called $\mathrm{H} / \alpha$ feature space. Upon the introduction of the aforementioned method, Cloude and Pottier suggested the partitioning of the plane in 9 regions, based on the polarimetric behaviour of known type of natural phenomenons. Therefore, once the $\mathrm{H}$ and the average $\alpha$ parameters are extracted from the target polarimetric signature, it is straightforward to classify it as one of the corresponding type of scattering mechanisms. Many works are based on such method, from geophysical parameters inversion algorithms (in varied regions from the globe) to detection and classification algorithms. Having a remarkable correspondence to ground truth, the usage of this unsupervised technique has had very few improvements since its conception. Nevertheless, there is an important remark still uncovered related to this method. The orthogonality constraint of the eigenvectors of the coherence matrix generates unfeasible regions in the plane. It is important to highlight that these regions are mathematically, and not physically, unfeasible.

Within this context, this paper addresses some of the main practical considerations when applying the independent component analysis (ICA) as an ICTD method with respect to both the H/ $\alpha$ and the TSVM [1] decompositions. 


\section{ICA BASED ICTD}

In [4], a new strategy to polarimetric target decomposition was presented by incorporating the ICA as an alternative to identify the canonical scattering mechanisms within an image cell. The ICA is a blind source separation technique based on higher order statistical moments and cumulants whose utility has already been explored in many different research areas, such as wireless communications, data compression and brain imaging applications. The results presented in [4] proved it to be a very promising area in polarimetry, mainly when nonGaussian heterogeneous clutters (inherent to high resolution SAR systems) are under study. The theoretical potential in estimating similar entropy and first component, when compared to traditional eigenvector decomposition, but rather a second most dominant component independent with respect to the first one and unconstrained by the orthogonality introduces an alternative way of physically interpreting a polarimetric SAR image. In [5], the ability of ICA to correctly identify clutters composed by non-orthogonal type of scatters is further investigated, as well as its performance under a sliding window approach, enabling a more accurate comparison to the results obtained with the Eigenvector based approach.

In general, the limiting curves on the $\mathrm{H} / \alpha$ plane reduce the analysis of high entropy type of targets in a more limiting way than the analysis of low entropy ones. Even though polarimetry is a better suited tool to analyse low entropy type of targets, the ability to correctly estimate the parameters that describe the illuminated scatters is crucial. Once again, it is important to highlight that these regions are mathematically, and not physically, unfeasible [6].

Previous results [5] demonstrate the $11 \times 11$ spatial neighbourhood is well suited for the estimation of the Touzi's TSVM parameters from the ICA mixing matrix (using Sigle Look Complex target vectors). This In the present work, our focus relies on addressing the following practical issue: to apply speckle filtering in ICA-ICTD for better preservation of spatial features.

\section{ICA-ICTD SPECKLE FILTERING}

One of the most powerful and widely used speckle filters in PolSAR is the Lee's sigma filter, with its recent improvement proposed in [8]. In this paper, we propose to use the same algorithm for deriving the adaptive MMSE coefficient $b$ from Eq. (6) in [8]. Further on, the following modifications are operated.

Firstly, instead of using each intensity image separately, we propose to use the Polarimetric Whitening Filter for taking into account simultaneously all the PolSAR channels. To do that, one good candidate is the texture parameter $\tau$ from [9] computed in each pixel:

$$
\widehat{\tau}=\mathbf{k}^{\dagger}[\widehat{M}]_{F P}^{-1} \mathbf{k}
$$

where $[\widehat{M}]_{F P}^{-1}$ is the Fixed Point polarimetric covariance matrix estimated over the centred boxcar neighbourhood and $\mathbf{k}$ the corresponding target vector.

Secondly, the MMSE filter is modified in each pixel of the PolSAR span image as:

$$
\widehat{s}=(1-b) \widehat{\sigma}_{0}+b \widehat{\tau}
$$

where $\widehat{\sigma_{0}}=\frac{\mathbf{k}^{\dagger}[\widehat{M}]_{F P}^{-1} \mathbf{k}}{\mathbf{k}^{\dagger}[\widehat{T}]_{S C M}^{-1} \mathbf{k}}$ and $[\widehat{T}]_{S C M}$ the sample covariance matrix.

Thirdly, the strong deterministic scatterers, previously detected by the 98 th percentile as in [8], are updating the values of the MMSE coefficient $b$ to 1 . For each pixel, the values of this coefficient is stored and will be used later for filtering the corresponding TSVM parameters. One can notice that, at this stage, we have available the filtered PolSAR span and the MMSE coefficient images.

When adapting the Lee's sigma filter to the ICA-ICTD, the most important constraint is the non-orthogonality of the ICA derived backscattering mechanisms. In [8], the MMSE filter from Eq. (2) has been directly applied for the multi-look PolSAR covariance matrix. This holds because two Sample Covariance estimates can be averaged, but this is not true for the ICA derived mixing matrices.

Instead of taking the average of the two covariance matrices, the mixing matrices barycenter could be addressed. The implementation of such approach is not straightforward, since the task of estimating matrix geometric means is a complex subject. The concept of geometric mean for more than two matrices has only been fully defined recently [10], powered by the association of the geometric mean of two positive definite matrices, $[M]_{1}$ and $[M]_{2}$, as the midpoint of the geodesic (with respect to a natural Riemannian metric) joining $[M]_{1}$ and $[M]_{2}$. While the derived theory is valid when addressing positive definite matrices (which is the case of the covariance matrices), it does not hold for the mixing matrices estimated with ICA, which are not necessarily positive definite.

Nevertheless, the solution relies on the rotational invariance of the Touzi's TSVM decomposition. From [1], one can observe that the rotation invariant scattering vector has the following form:

$$
\overrightarrow{\mathbf{v}}^{\text {orient-inv }}=\mu\left[\begin{array}{c}
\cos \alpha_{s} \cos \left(2 \tau_{m}\right) \\
\sin \alpha_{s} e^{j \Phi_{\alpha_{s}}} \\
-j \cos \alpha_{s} \sin \left(2 \tau_{m}\right)
\end{array}\right],
$$

where the four roll-invariant parameters $\alpha_{s}$ (symmetric scattering type magnitude), $\Phi_{\alpha_{s}}$ (symmetric scattering type phase), $\tau_{m}$ (helicity), and $\mu$ (maximum amplitude return) are necessary for an unambiguous description of the corresponding PolSAR scattering mechanism. As described in [1], $\overrightarrow{\mathbf{V}}$ orient-inv is obtained by constructing the Graves power matrix and performing the con-diagonalization from [11] followed by the Huynen desying. 
In each pixel, we propose to filter independently each rotation invariant scattering vector $\overrightarrow{\mathbf{v}}_{i}^{\text {orient-inv }}$ retrieved from the columns of the ICA derived mixing matrix $(i=\overline{1,3}$ for monostatic PolSAR). The two vector estimates $\overrightarrow{\mathbf{v}}_{3 \times 3}^{\text {orient-inv }}$ and $\overrightarrow{\mathbf{v}}_{11 \times 11}^{\text {orientinv }}$ correspond to the ICA applied independently on $3 \times 3$ and respectively $11 \times 11$ boxcar neighbourhoods:

$$
\overrightarrow{\mathbf{v}} \text { orient }-i n v=(1-b) \overrightarrow{\mathbf{v}}_{11 \times 11}^{\text {orient-inv }}+b \overrightarrow{\mathbf{v}}_{3 \times 3}^{\text {orient-inv }} .
$$

Finally, the Cloude and Pottier entropy can be evaluated using the maximum amplitude returns $\mu_{i}$, while the Cloude and Pottier $\alpha$ is equivalent to Touzi's $\alpha_{s}$ for symmetric scattering [1].

\section{POLSAR EXPERIMENTAL RESULTS}

The PolSAR dataset was acquired by the French Aerospace Lab (ONERA), in 2009, over the French Guiana, in the frame of the ESA campaign TropiSAR. Fig. 1-(a),(b) shows the classification results obtained using the $\mathrm{H} / \alpha$ eigenvector decomposition (PCA) and $\mathrm{H} / \alpha$ ICA decomposition, respectively. One can notice that similar mechanisms were detected, mostly in zone 2, with significantly less bias in the ICA based ICTD.

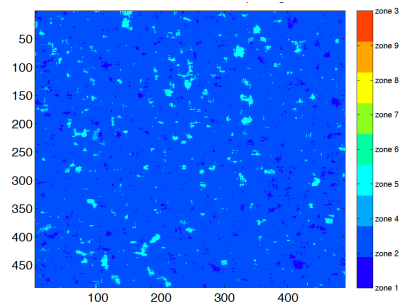

(a)

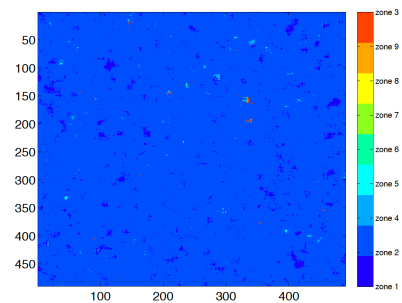

(b)
Fig. 1. Paracou P-band airborne dataset, $H / \alpha$ classification map: (a) PCA and (b) ICA.

The PolSAR speckle filtering results are illustrated in Fig. 2-(a),(b). In this paper, a decimation by a factor 2 has been applied both in range and azimuth (equivalent to a 4-look covariance matrix). As expected, the proposed MMSE filter outperforms the boxcar filter in terms of spatial resolution preservation.

Fig. 4-(a),(b),(c),(d) shows the Touzi's roll invariant TSVM parameters computed by ICA MMSE speckle filtering, as compared to the ones obtained by applying the boxcar filter and PCA from Fig. 3-(a),(b),(c),(d).

Finally, we propose to analyse the results by representing the derived TSVM parameters of symmetric targets on the Poincare sphere (helicity equal 0). It can be observed in Fig. 5-(a),(b) that the second and the third most dominant mechanism (represented in blue and green, respectively) occupy different position onto the sphere, thus meaning that the

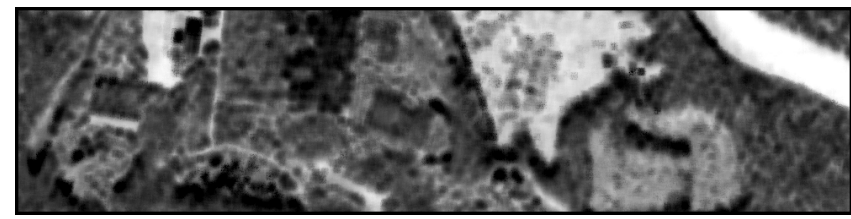

(a)

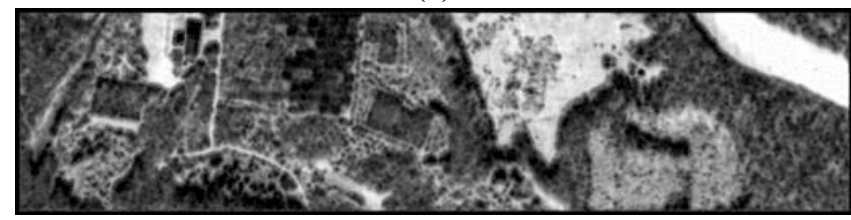

(b)

Fig. 2. Paracou P-band airborne dataset, speckle filtering results, filtered PolSAR span image (in dB) : (a) boxcar and (b) MMSE.

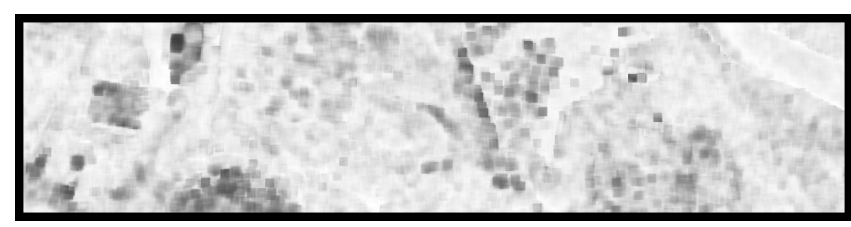

(a)

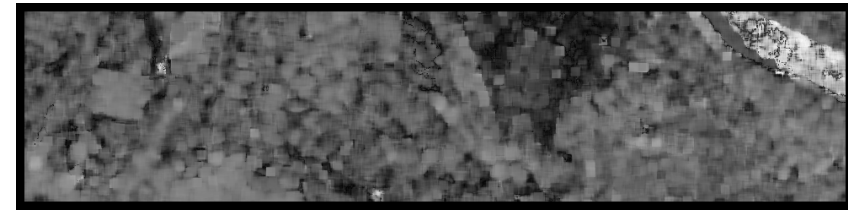

(b)

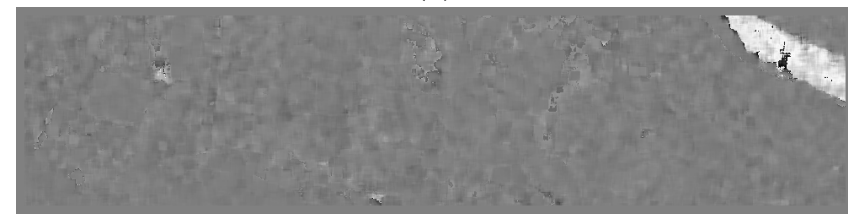

(c)

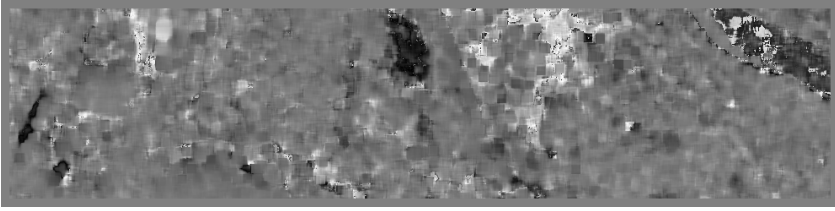

(d)

Fig. 3. Paracou P-band airborne dataset, TSVM parameters after PCA boxcar speckle filtering: (a) entropy, (b) symmetric scattering type magnitude, (c) helicity and (d) symmetric scattering type phase. 


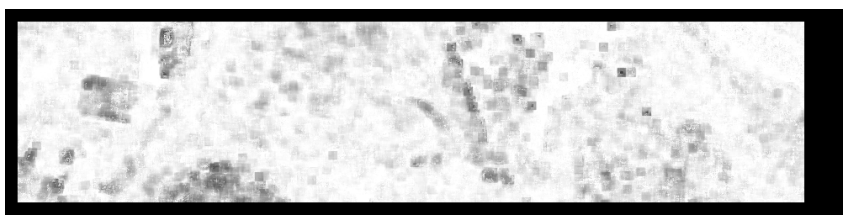

(a)

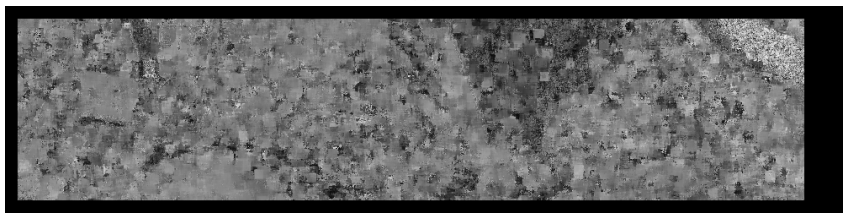

(b)

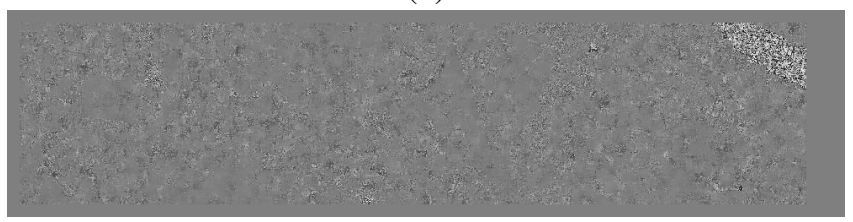

(c)

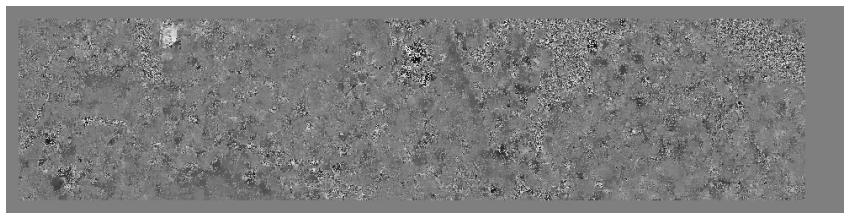

(d)

Fig. 4. Paracou P-band airborne dataset, TSVM parameters after ICA MMSE speckle filtering: (a) entropy, (b) symmetric scattering type magnitude, (c) helicity and (d) symmetric scattering type phase.

non-orthogonality of ICA will produce different mechanisms, indeed.

\section{CONCLUSION}

This paper addressed one important practical aspect when computing the ICA-ICTD on real PolSAR data: speckle filtering. By applying the MMSE filter on each of the ICA derived rotation invariant scattering vectors, we demonstrated the spatial resolution can be better preserved with respect to the conventional PolSAR boxcar speckle filter. Further studies will address plugging the ICA-ICTD in a reliable PolSAR segmentation algorithm.

\section{REFERENCES}

[1] R. Touzi, "Target scattering decomposition in terms of rollinvariant target parameters," IEEE Transactions on Geoscience and Remote Sensing, vol. 45, no. 1, pp. 73-84, 2007.

[2] W. L. Cameron and L. K. Leung, "Feature motivated polarization scattering matrix decomposition," in Proceedings of the IEEE International Radar Conference, Arlington, USA, 1990, pp. 549-557.

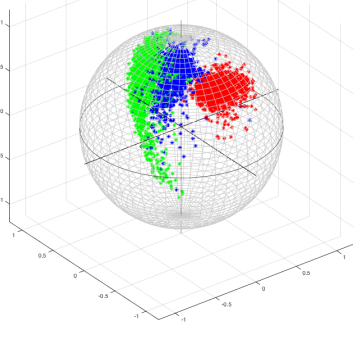

(a)

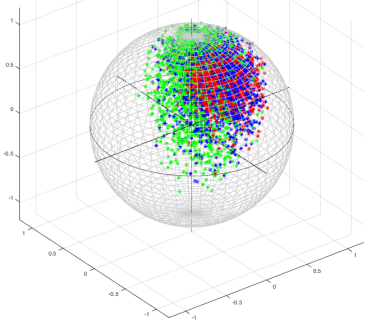

(b)
Fig. 5. Paracou P-band airborne dataset, Poincaré sphere representation of symmetric targets: (a) PCA with boxcar, (b) MMSE with ICA.

[3] S. R. Cloude and E. Pottier, "A review of target decomposition theorems in radar polarimetry," IEEE Transactions on Geoscience and Remote Sensing, vol. 32, no. 6, pp. 498-518, 1996.

[4] N. Besic, G. Vasile, J. Chanussot, and S. Stankovic, "Polarimetric incoherent target decomposition by means of independent component analysis," IEEE Transactions on Geoscience and Remote Sensing, vol. 53, no. 3, pp. 1236-1247, 2015.

[5] L. Pralon, G. Vasile, M. Dalla-Mura, J. Chanussot, and N. Besic, "Evaluation of ICA-based ICTD for PolSAR data analysis using a sliding window approach: convergence rate, gaussian sources, and spatial correlation," IEEE Transactions on Geoscience and Remote Sensing, vol. 54, no. 7, pp. 42624271, 2016.

[6] L. Pralon, G. Vasile, M. Dalla-Mura, and J. Chanussot, "Evaluation of the new information in the $\mathrm{H} / \alpha$ feature space provided by ICA in PolSAR data analysis," IEEE Transactions on Geoscience and Remote Sensing, vol. 55, no. 12, pp. 6893-6909, 2017.

[7] S. R. Cloude and E. Pottier, "An entropy based classification scheme for land applications of polarimetric SAR," IEEE Transactions on Geoscience and Remote Sensing, vol. 35, no. 1, pp. 68-78, 1997.

[8] J. S. Lee, T. L. Ainsworth, Y. Wang, and K. S. Chen, "Polarimetric SAR speckle filtering and the extended sigma filter," IEEE Transactions on Geoscience and Remote Sensing, vol. 53, no. 3, pp. 1150-1160, 2015.

[9] G. Vasile, F. Pascal, J.-P. Ovarlez, P. Formont, and M. Gay, "Optimal parameter estimation in heterogeneous clutter for high resolution polarimetric SAR data," IEEE Geoscience and Remote Sensing Letters, vol. 8, no. 6, pp. 1046-1050, 2011.

[10] R. Bhatia and J. Holbrook, "Riemannian geometry and matrix geometric means," Linear Algebra and its applications, vol. 413, no. 2, pp. 594-618, 2005.

[11] A. Kostinski and W. M. Boerner, "On foundations of radar polarimetry," IEEE Transactions on Antennas and Propagation, vol. 34, no. 12, pp. 1395-1404, 1986. 